\title{
HFE-associated hereditary hemochromatosis
}

\author{
Jacob Alexander, $M D^{I}$, and Kris V. Kowdley, $M D^{1,2,3}$
}

TABLE OF CONTENTS

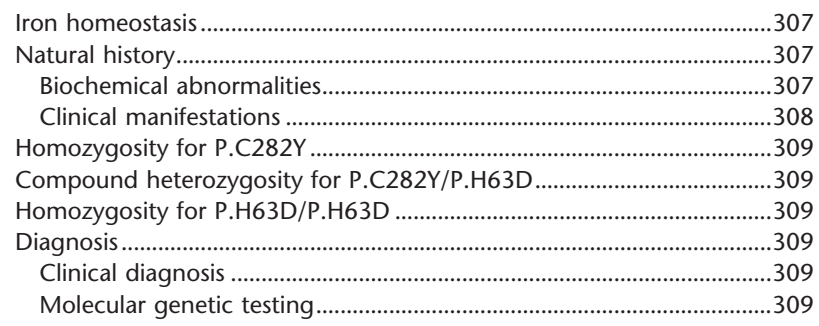

Abstract. In populations of northern European descent, the p.C282Y mutation in the HFE gene is highly prevalent, and HFE-associated hereditary hemochromatosis is the most common type of inherited iron overload disorder. Inappropriate low secretion of hepcidin, which negatively regulates iron absorption, is postulated to be the mechanism for iron overload in this condition. The characteristic biochemical abnormalities are elevated serum transferrin-iron saturation and serum ferritin. Typical clinical manifestations include cirrhosis, liver fibrosis, hepatocellular carcinoma, elevated serum aminotransferase levels, diabetes mellitus, restrictive cardiomyopathy and arthropathy of the second and third metacarpophalangeal joints. Most patients are now diagnosed before the development of these clinical features. Molecular genetic tests are currently available for genotypic diagnosis. In selected individuals, diagnosis might require liver biopsy or quantitative phlebotomy. Iron depletion by phlebotomy is the mainstay of treatment and is highly effective in preventing the complications of iron overload if instituted before the development of cirrhosis. Genetic testing is currently not recommended for population screening because of low yield as the majority of the healthy, asymptomatic p.C282Y homozygotes do not develop clinically significant iron overload. HFE gene testing remains an excellent tool for the screening of first-degree relatives of affected probands who are p.C282Y homozygotes. Genet Med 2009:11(5): 307-313.

Key Words: hemochromatosis, HFE gene, iron overload, phlebotomy

$\mathrm{H}$ ereditary hemochromatosis $(\mathrm{HH})$ is an inherited disorder characterized by progressive iron deposition and tissue injury in multiple organs secondary to an inherited predisposition to excessive and inappropriately regulated intestinal iron absorption. ${ }^{1,2} \mathrm{HH}$ has been demonstrated to result from mutations in several genes involved in the regulation of iron ho-

From the ${ }^{1}$ Department of Medicine, and ${ }^{2}$ Division of Gastroenterology, University of Washington; and ${ }^{3}$ Center for Liver Disease, Digestive Disease Institute, Virginia Mason Medical Center, Seattle, Washington.

Kris V. Kowdley, MD, Benaroya Research Institute, 1201 Ninth Avenue, MS IN-RC, Seattle, WA 98101-2795. E-mail: KrisK@medicine.washington. edu.

Disclosure: The authors declare no conflicts of interest.

Submitted for publication December 30, 2008.

Accepted for publication January 16, 2009.

DOI: $10.1097 /$ GIM.0b013e31819d30f2

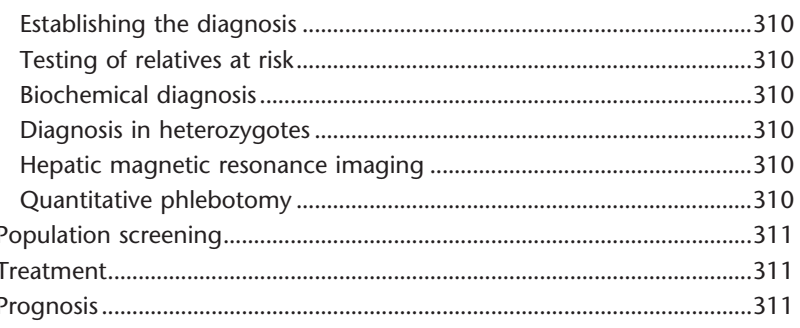

meostasis such as HFE, TfR2, HJV, HAMP, and Ferroportin. ${ }^{3}$ The most common form of HH is associated with the homozygous p.C282Y mutation of the HFE gene, which was first identified by Feder et al. ${ }^{4}$ by positional cloning in 1996. Although the prevalence of end-organ damage secondary to HFEassociated $\mathrm{HH}$ (HH-HFE) is relatively low, the p.C282Y mutation is highly prevalent, with a prevalence of $1: 200$ to $1: 250$ for p.C282Y homozygozity and 1:8 to $1: 12$ for p.C282Y heterozygozity among populations of northern European ancestry. ${ }^{5}$ This review focuses on the natural history, diagnosis, and treatment of HH-HFE.

\section{IRON HOMEOSTASIS}

In normal adults, iron loss and iron absorption are in balance, each amounting to approximately $1 \mathrm{mg}$ /day in men, and $1.5-2$ $\mathrm{mg}$ /day in women. ${ }^{6}$ The total body iron content in adult men is approximately $35-45 \mathrm{mg} / \mathrm{kg}$ of body weight, with lower levels in premenopausal women secondary to menstrual loss. ${ }^{6}$ Erythrocyte hemoglobin comprises more than two thirds of the iron pool, with storage iron in the liver accounting for most of the rest. ${ }^{6}$ Increased metabolic demand for iron results in increased intestinal iron absorption and mobilization of iron from tissue stores; the reverse happens in conditions of decreased iron demand. ${ }^{6}$ Sloughing of intestinal mucosal cells and menstrual blood loss are the main physiological processes responsible for loss of iron from the body. ${ }^{6}$ Because there are no regulatory mechanisms to control these processes according to body iron status, regulation of the amount of iron in the body takes place mainly at the level of absorption. ${ }^{7}$

A schematic representation of normal iron regulation and the abnormalities in $\mathrm{HH}$ is given in Figure $1 .{ }^{8}$ Defective hepcidin synthesis in the liver is postulated to be the central pathogenic factor in HH-HFE. However, the link between $H F E$ gene mutations and defective hepcidin synthesis has not yet been elucidated. ${ }^{9}$

\section{NATURAL HISTORY}

\section{Biochemical abnormalities}

Elevation of serum transferrin-iron saturation, in association with increased nontransferrin-bound iron is the first biochemical abnormality in $\mathrm{HH}$. This is followed by elevation of serum 

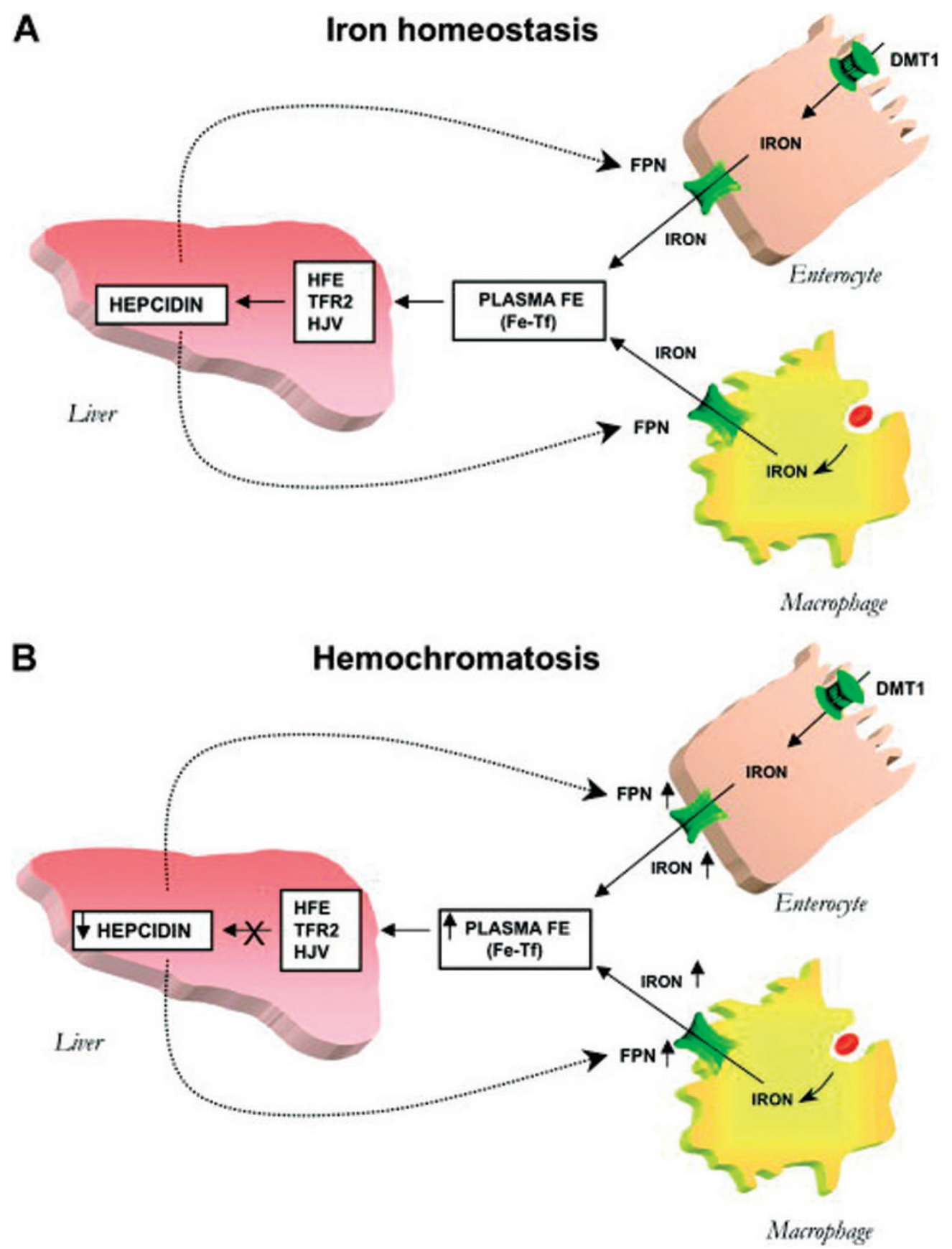

Fig. 1. Iron regulation. A, Under normal conditions, plasma transferrin saturation regulates the expression of liver hepcidin via a HFE, TFR2, and BMP-HJV signaling pathway, is secreted into the blood, binding to FPN in the intestine and macrophages inducing FPN internalization and degradation, limiting intestinal Fe absorption and Fe recycling by macrophages to maintain plasma transferrin saturation. B, In HH, mutations in HFE, HJV, and TFR2 impair hepcidin synthesis, increasing FPN levels and Fe release from intestinal cells and macrophages, elevating plasma transferrin saturation and causing deposition of iron in the liver and other tissues. Reprinted with permission of John Wiley \& Sons, Inc. Olynyk JK, Trinder D, Ramm GA, Britton RS, Bacon BR. Hereditary hemochromatosis in the post-HFE era. Hepatology 2008;48(3):991-1001. Copyright (C) 2008 by the American Association for the Study of Liver Disease.

ferritin, which reflects iron overload in parenchymal cells. Because serum iron is high, and as the iron transport to bone marrow and incorporation into hemoglobin are unaffected, erythropoiesis is characteristically unimpaired in $\mathrm{HH}^{2}$

\section{Clinical manifestations}

Progressive tissue iron overload and clinical complications occur only in a minority of p.C282Y homozygotes. ${ }^{10-12}$ Genetic and environmental factors influencing iron absorption and stor- 
age have been implicated for the low penetrance of the p.C282Y mutation. The risk of developing clinical complications depends primarily on the degree of iron loading. Environmental factors that increase the risk of cirrhosis include excess alcohol consumption, steatosis, and coexistent viral hepatitis. ${ }^{13}$ Previous reports have suggested that men are 10 times more likely than women to have symptoms of organ failure resulting from $\mathrm{HH}$ $H F E,{ }^{10-12}$ but recent studies have shown that among individuals with HH-HFE, women are up to half as likely as men to develop complications such as cirrhosis and diabetes. ${ }^{14,15}$ Genetic factors such as polymorphisms in genes associated with hepatic fibrogenesis, antioxidant activity, and inflammation are being evaluated as potential modifiers of the risk of cirrhosis in p.C282Y homozygotes. ${ }^{16}$

The clinical manifestations of HH- $H F E$ depend on the degree of parenchymal iron accumulation. Although this relationship is not linear and clinical expression in individual patients is highly variable, organ damage is generally observed with $>20 \mathrm{~g}$ of parenchymal iron storage. ${ }^{17}$ Clinical manifestations include hepatomegaly, cirrhosis, arthropathy involving the metacarpophalangeal joints, progressive increase in skin pigmentation, diabetes mellitus, and cardiomyopathy. ${ }^{17}$ Other common but less specific manifestations include abdominal pain, weakness, lethargy, and weight loss. ${ }^{18}$ Men may develop impotence from hypogonadotropic hypogonadism secondary to pituitary involvement. ${ }^{19}$

The major causes of death in HH-HFE are decompensated cirrhosis, hepatocellular carcinoma, diabetes mellitus, and cardiomyopathy. Compared with the age- and sex-matched population without $\mathrm{HH}-H F E$, the risk of cardiomyopathy is 306 times higher and that of cirrhosis is 13 times higher in patients with HH-HFE. ${ }^{20}$ The reported estimates of the risk of HCC in patients with HH-HFE vary from 20 to 219 times that of the general population. ${ }^{15,21-24}$ This wide variation in the reported risk of HCC has been argued to be the result of the heterogeneity of the patient population arising from referral bias in different centers. ${ }^{25}$ Among patients with $\mathrm{HH}-H F E$, the increased risk of HCC occurs predominantly in those with cirrhosis. ${ }^{25,26}$

\section{HOMOZYGOSITY FOR P.C282Y}

Earlier studies suggested that the penetrance of homozygosity for p.C282Y was relatively high; however, subsequent studies have claimed that clinically significant disease may develop in as few as $2 \%$ of p.C $282 \mathrm{Y}$ homozygotes. ${ }^{27}$ This heterogeneity in the estimates of penetrance is the result of the use of different criteria for the definition of penetrance and lack of comparison with matched controls in older studies. ${ }^{28}$ Prevalence of the clinical manifestations of HH-HFE such as joint pain, arthritis, skin pigmentation, and infertility have recently been found to be similar among screened p.C282Y homozygotes compared with the general population. ${ }^{27,29}$ Morbidity and quality of life have also been found to be similar in p.C282Y homozygotes compared with the general population. ${ }^{30}$ Based on these observations, it has been concluded that the penetrance for clinical disease is low. ${ }^{30}$ However, there is no currently available test to predict whether a p.C282Y homozygote will develop clinical disease. ${ }^{18}$

\section{COMPOUND HETEROZYGOSITY FOR P.C282Y/P.H63D}

The penetrance of p.C282Y/p.H63D genotype is lower than for p.C282Y homozygotes, with only about $0.5-2.0 \%$ of patients developing clinical signs of iron overload. Many
p.C282Y/p.H63D compound heterozygotes that develop clinical evidence of iron overload seem to have a complicating factor such as fatty liver or viral hepatitis. ${ }^{18}$

\section{HOMOZYGOSITY FOR P.H63D/P.H63D}

The p.H63D/p.H63D genotype has an even lower penetrance than the p.C282Y/p.H63D genotype. Although this mutation is associated with a significant increase in serum transferrin-iron saturation, it is generally not associated with clinically significant iron overload. ${ }^{31}$

\section{DIAGNOSIS}

With the widespread application of biochemical and genotypic tests, patients are being increasingly identified in the presymptomatic stage, either through detection of abnormal iron-related studies or by family screening. ${ }^{18}$

\section{Clinical diagnosis}

The diagnosis of HH-HFE is often suspected when patients present with features such as cirrhosis, liver fibrosis, hepatocellular carcinoma, elevated serum aminotransferase levels, restrictive cardiomyopathy, and arthropathy of the second and third metacarpophalangeal joints. Currently, the majority of cases of HH-HFE are diagnosed after evaluation of elevated serum transferrin-iron saturation and serum ferritin concentration. Occasional patients are identified after evaluation for elevated serum aminotransferases or symptoms such as lethargy, arthralgia, abdominal pain, and/or decreased libido. ${ }^{18}$

\section{Molecular genetic testing}

Testing for the common mutations in the $H F E$ gene (p.C282Y, p.H63D, and p.S65C) is now widely available. ${ }^{32}$ Because of incomplete penetrance and the presence of multiple genetic and environmental factors influencing the expression of clinical outcomes, molecular genetic testing is not an effective test for population screening. It is of potential use in the following clinical situations: ${ }^{18}$

- Confirmatory diagnostic testing

- Predictive testing for at-risk relatives

- Carrier testing (for the identification of heterozygotes)

- Prenatal diagnosis (technically available but rarely performed)

The two methods of molecular genetic testing are targeted mutation analysis and gene sequence analysis. ${ }^{18}$ Targeted mutation analysis involves testing for one or more specific mutations and is available on a clinical basis for p.C282Y and p.H63D alleles which together account for about $86 \%$ of individuals of European origin with HH-HFE (p.C282Y homozygotes accounting for $80 \%$ and p.C282Y/ p.H63D compound heterozygotes accounting for $6 \%) .{ }^{18}$ Because p.C282Y/p.S65C and p.H63D/p.S65C heterozygotes together account for only $1 \%$ of individuals with clinically significant $\mathrm{HH}-H F E$, most clinical laboratories do not routinely test for the p.S65C allele. ${ }^{33}$ Sequence analysis involves sequencing one or more segments of DNA to identify variations. ${ }^{18}$ Although targeted mutation analysis identifies only the specific targeted mutations, sequence analysis identifies any variation from the wild type of the $H F E$ gene, and is useful to identify the other less common mutant alleles associated with HH-HFE. However, sequence analysis is more expensive and is available only in a limited number of clinical and research laboratories. ${ }^{18}$ 


\section{Establishing the diagnosis}

Targeted mutation analysis is indicated in adults with transferrin-iron saturation higher than $45 \%$ (Fig. 2). Presence of p.C282Y homozygozity or p.C282Y/p.H63D compound heterozygozity is diagnostic of HH-HFE in these individuals. ${ }^{18}$ Absence of these mutations generally indicate iron overload arising from other causes, including liver disease unrelated to HH-HFE. Evaluation of such patients requires liver biopsy for histological examination and estimation of hepatic iron concentration. ${ }^{34,35}$

\section{Testing of relatives at risk}

The commonly followed strategy for testing the adult relatives of probands is as follows:

Step 1-Molecular genetic testing to identify p.C282Y homozygous siblings of probands homozygous for p.C282Y.

Step 2-Iron studies for those who are found to be p.C282Y homozygotes.

\section{Biochemical diagnosis}

The biochemical markers of HH-HFE are elevated serum transferrin-iron saturation and/or elevated serum ferritin concentration. Elevation of serum transferrin-iron saturation is the earliest phenotypic abnormality and is a reliable indicator of risk of the iron overload in HH-HFE; the level is not age-related in adults and does not correlate with the presence or absence of symptoms. ${ }^{18,36}$ About $80 \%$ of individuals with HH-HFE have had a fasting transferrin-iron saturation of at least $60 \%$ (men) or at least $50 \%$ (women) on two or more occasions in the absence of other known causes of elevated transferrin-iron saturation. ${ }^{18}$ A threshold transferrin-iron saturation of $45 \%$ has been proposed as more sensitive than the higher values used in the past for detecting $\mathrm{HH}-H F E$ but it could be less specific as it may identify heterozygotes who are not at risk of developing clinical disease. ${ }^{36}$ In addition, when applied to young adults, this threshold could fail to detect up to $60 \%$ of p.C282Y homozygotes who subsequently develop elevated serum transferrin-iron saturation. ${ }^{37}$ Therefore, sequential measurements over a period of several years may be required to confirm nonexpression in young adults.

Serum ferritin concentration correlates with total body iron stores and hepatic iron concentration in patients with $\mathrm{HH}$ $H F E .{ }^{38,39}$ Additionally, serum ferritin has been shown to be a

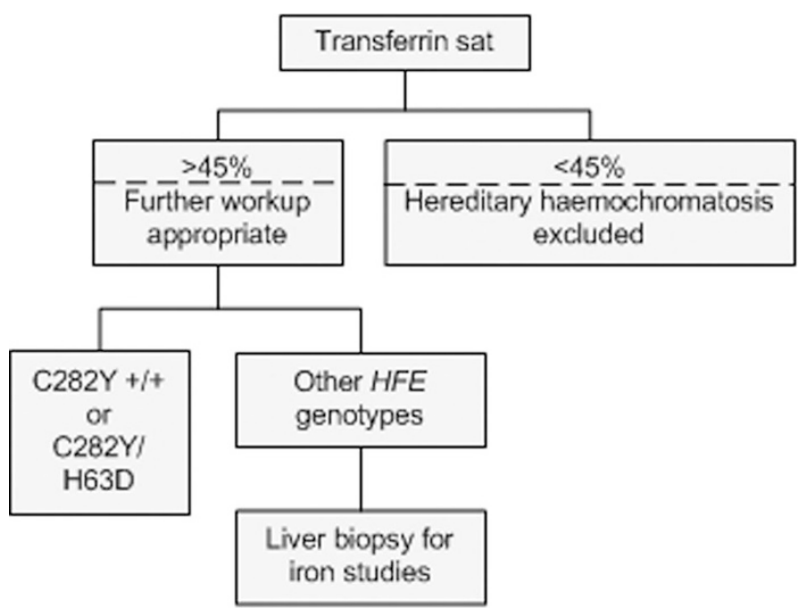

Fig. 2. Algorithm for the diagnosis of HH-HFE. highly sensitive predictor of cirrhosis in HH-HFE, with the occurrence of cirrhosis restricted almost completely to those with serum ferritin levels $>1000 \mu \mathrm{g} / \mathrm{L} .{ }^{40-42}$ Because serum ferritin accurately identifies individuals at increased risk for cirrhosis, it has recently been argued that screening with serum ferritin alone may be sufficient to detect clinically significant disease. ${ }^{43}$ A nomogram incorporating a wide range of serum ferritin and transferrin-iron saturation values has been developed in an attempt to estimate the probability of HH- $H F E$ based on serum ferritin and transferrin-iron saturation, but this has not been widely adopted. ${ }^{4}$

However, elevated serum ferritin concentration may lack specificity for iron overload as it is an acute phase reactant and is induced by inflammatory cytokines in the presence of inflammation, hepatocellular necrosis, and malignancy. Additionally, hyperferritinemia is associated with secondary iron overload syndromes, excessive alcohol consumption, nonalcoholic fatty liver disease, chronic viral hepatitis, alpha-1 antitrypsin deficiency, autoimmune liver diseases, and Wilson disease. ${ }^{45,46}$ Clinical assessment, HFE genotyping, and other blood tests generally help to distinguish these conditions from HH. ${ }^{46}$ Quantification of hepatic iron content by magnetic resonance imaging has also been found to be useful in making this distinction; hepatic iron content $>3$ times the upper limit of normal is highly unlikely in the absence of $\mathrm{HH}$ or secondary iron overload $^{46}$ In selected situations, liver biopsy may be required to distinguish $\mathrm{HH}$ from other causes of hyperferritinemia. ${ }^{46}$

\section{Diagnosis in heterozygotes}

Studies suggest that some overlap occurs in serum transferrin-iron saturation level among homozygotes and heterozygotes. In one study, $2 \%$ of male heterozygotes had a fasting transferrin-iron saturation above $62 \%$, and $3 \%$ of female heterozygotes had a fasting transferrin-iron saturation above $50 \% .{ }^{36}$ Twenty percent of male heterozygotes and $8 \%$ of female heterozygotes have serum ferritin concentrations that exceed the 95th percentile value for age-matched controls. ${ }^{18}$ However, the abnormalities in iron studies observed in p.C282Y heterozygotes generally do not predict clinically important body iron overload in the absence of another genetic or environmental cofactor. ${ }^{18,47}$

p.C282Y/p.H63D subjects generally have elevated iron indices, but the risk of developing progressive liver disease is low in the absence of comorbid factors such as steatosis, diabetes, or excess alcohol consumption. ${ }^{48}$

\section{Hepatic magnetic resonance imaging}

A new magnetic resonance imaging technique has been developed to noninvasively quantify hepatic iron content by using the paramagnetic properties of iron. ${ }^{49}$ This technique is based on the measurement and imaging of proton transverse relaxation rates within the liver. ${ }^{49}$

This technique has been found to be accurate and precise over a wide range of liver iron concentrations and allows for assessment of the regional variation of hepatic iron content in addition to the average concentration.

\section{Quantitative phlebotomy}

In individuals with biochemical evidence of iron overload who are not p.C282Y homozygotes and are unable or unwilling to undergo liver biopsy, quantitative phlebotomy can be used to determine the quantity of iron that can be mobilized, thus confirming the diagnosis of parenchymal iron overload. ${ }^{18}$ Each phlebotomy removes approximately $500 \mathrm{~mL}$ of blood, amounting to 250 $\mathrm{mg}$ of iron in persons with normal hematocrit. ${ }^{50}$ The amount of 


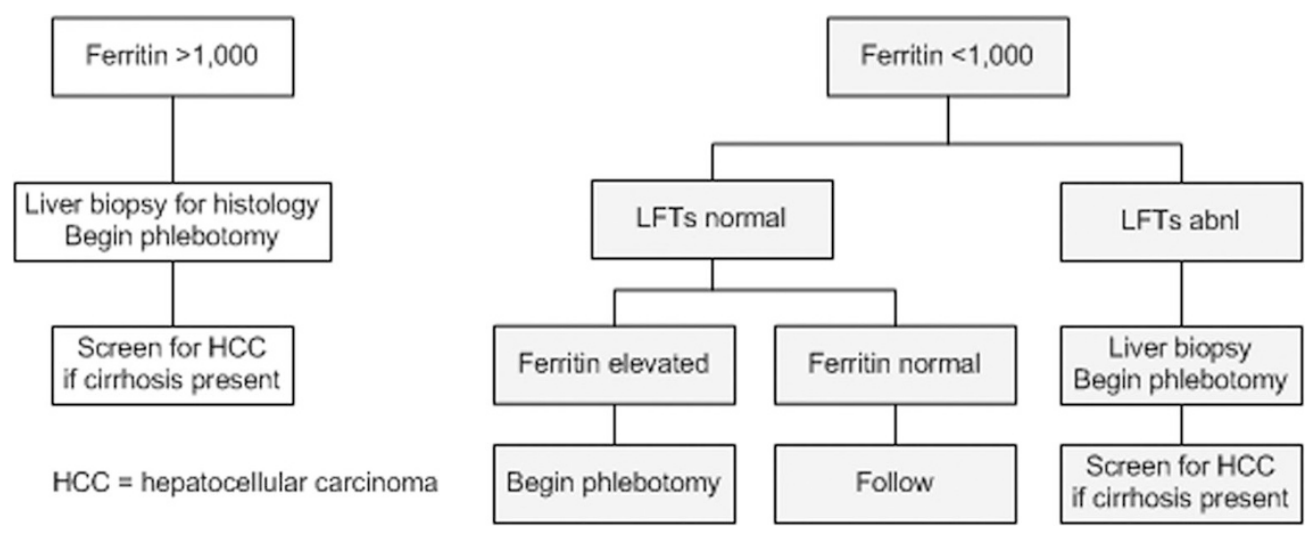

Fig. 3. Algorithm for the management of HH-HFE.

iron removed can be calculated by multiplying the number of phlebotomies with 0.25 ; but this number needs to be adjusted when hematocrit is abnormal. In the absence of causes of secondary iron overload, removal of $4 \mathrm{~g}$ or more of mobilizable iron stores before the onset of iron-limited erythropoiesis confirms the presence of hemochromatosis. ${ }^{50}$ Most individuals fully expressing the phenotype have more than $4 \mathrm{~g}$ of mobilizable iron. ${ }^{18}$

\section{POPULATION SCREENING}

Population screening for HH-HFE has been controversial; studies documenting the low penetrance of the disease have been cited in the argument against population screening, whereas studies demonstrating a more substantial risk for organ damage have been cited in the argument in support of population screening. ${ }^{32}$ Recently, a comprehensive cost analysis showed that although population-based screening is not significantly more cost efficient than not screening, it is associated with the increase of 7 years in the life expectancy of individuals identified with $\mathrm{HH}-H F E$ and treated. ${ }^{51}$ In this analysis, genetic testing was found to be the most cost effective as the final confirmatory test, rather than as the initial screening test. Additionally, concerns of psychological harm and genetic discrimination surround the use of genotypic test for population screening. ${ }^{32}$ Therefore, phenotypic screening with serum transferrin-iron saturation test (serum iron divided by serum total iron-binding capacity) or unsaturated iron-binding capacity has been proposed as the optimal strategy for screening. ${ }^{32}$ Although there are some concerns about false-negative and false-positive results, phenotypic screening seems reasonable in screening adult men (age $\geq 25$ years) of Northern European ancestry. ${ }^{32}$

\section{TREATMENT}

Iron depletion with phlebotomy is the mainstay of treatment of HH-HFE. The number of phlebotomies required to achieve iron depletion varies depending on the degree of iron overload. In the induction phase, one unit of blood (containing approximately $250 \mathrm{mg}$ of iron) is removed once a week until the serum ferritin level is $<50 \mathrm{ng} / \mathrm{mL}$; the frequency of phlebotomy may need to be decreased if the erythropoetic response is blunted (i.e., if there is a reduction in hemoglobin to $<11 \mathrm{~g} / \mathrm{dL}$ ). Serum ferritin is checked once a month until it is below the upper limit of normal (i.e., $300 \mathrm{ng} / \mathrm{mL}$ in men and $200 \mathrm{ng} / \mathrm{mL}$ in women); thereafter, it serum ferritin should be measured twice monthly. ${ }^{52}$
The duration of initial induction therapy may last weeks to months or may take up to 2 or 3 years. Once the target of iron depletion is reached, the frequency of phlebotomy is greatly reduced and maintenance treatment can be performed as needed. Before each venesection, the hematocrit is measured and phlebotomy is postponed if the hematocrit is $<32$. Once in every $10-12$ phlebotomies, serum ferritin should be checked; a serum ferritin level $<25 \mu \mathrm{g} / \mathrm{L}$ indicates an iron deficient state and mandates temporary withholding of further phlebotomies. Although malaise, fatigue, skin pigmentation, insulin requirements in diabetes and abdominal pain associated with $\mathrm{HH}-H F E$ have been shown to improve with phlebotomy, arthropathy, hypogonadism, and cirrhosis are less responsive to iron removal.

Surveillance for hepatocellular carcinoma has been recommended in HH-HFE patients with cirrhosis. ${ }^{17}$ This is commonly performed with biannual abdominal ultrasound examination and/or computed tomography scan and measurement of serum AFP concentration. Avoidance of alcohol, medicinal iron, mineral supplements, excess Vitamin C, and uncooked seafood is also recommended in all individuals with $\mathrm{HH}-H F E .{ }^{18}$

\section{PROGNOSIS}

Prognosis of HH-HFE depends on the degree and duration of iron overload. ${ }^{53}$ Most individuals identified on the basis of homozygozity for the p.C282Y mutation do not have evidence of significant organ damage such as advanced cirrhosis, cardiac failure, skin pigment changes, or diabetes. ${ }^{18}$ These individuals have a low risk of progression to clinical disease and have an expected survival similar to that of the general population. ${ }^{27,54,55}$ However, individuals with elevated hepatic iron concentrations have an increased risk of clinical manifestations such as diabetes, cirrhosis, and cardiac disease. ${ }^{56}$ The survival of individuals with end-organ damage is lower than age- and sex-matched normal populations, with the presence of cirrhosis being the primary determinant of survival. ${ }^{53,55,57}$

The risk factors for hepatocellular carcinoma in patients with HH- HFE are presence of cirrhosis, severity, and duration of iron overload, advanced age, alcoholism, tobacco smoking, and concomitant infection with Hepatitis C or Hepatitis B. ${ }^{26}$ The occurrence of hepatocellular carcinoma is generally limited to patients with cirrhosis, but a number of cases have been reported in patients with HH-HFE, where hepatocellular carcinoma has developed in the absence of cirrhosis. ${ }^{58}$ Primary nonhepatocellular malignancies such as colorectal cancer, gastric carcinoma, prostate cancer, breast 
cancer, acute myelogenous leukemia, Hodgkin lymphoma, and non-Hodgkin lymphoma have also been reported in association with $\mathrm{HH}-H F E .{ }^{59}$ However, in contrast to the well-established causal association between HH-HFE and hepatocellular carcinoma, the association of $\mathrm{HH}-H F E$ with nonhepatocellular malignancies has not been clearly established. ${ }^{59}$

Patients who develop biochemical evidence of iron overload in whom phlebotomy is started and adequate iron depletion is achieved before the onset of cirrhosis or diabetes have been found to have survival comparable with that of the general population. ${ }^{53-55,57}$ Survival is decreased for those individuals who have cirrhosis at the time of initiation of iron depletion therapy. In addition, iron depletion therapy does not eliminate the 10-30\% risk for hepatocellular carcinoma and cholangiocarcinoma in patients with cirrhosis. The common causes of death in those who have developed end organ damage are hepatic failure, hepatocellular carcinoma, congestive heart failure, and cardiac arrhythmias. It has not yet been clearly determined whether progressive iron overload occurs in p.C282Y homozygotes with elevated serum transferrin-iron saturation and serum ferritin concentrations with no evidence of overt end-organ damage. Therefore, the prognosis of this group of individuals is more difficult to ascertain. The available evidence suggests that although serum ferritin concentration may rise over time, the risk of developing clinically significant end-organ damage is very low. ${ }^{37,54,60}$

\section{ACKNOWLEDGMENTS}

Supported in part by NIH Grant DK-02957 (to K.V.K.).

\section{REFERENCES}

1. Finch SC, Finch CA. Idiopathic hemochromatosis, an iron storage disease. A. Iron metabolism in hemochromatosis. Medicine (Baltimore) 1955;34:381-430.

2. Pietrangelo A. Hemochromatosis: an endocrine liver disease. Hepatology 2007;46:1291-1301

3. Online Mendelian Inheritance in Man, OMIM. McKusick VA, editor. Available at http://www.ncbi.nlm.nih.gov/entrez/dispomim.cgi?id=235200. Accessed November 10, 2008

4. Feder JN, Gnirke A, Thomas W, et al. A novel MHC class I-like gene is mutated in patients with hereditary haemochromatosis. Nat Genet 1996;13: $399-408$

5. Merryweather-Clarke AT, Pointon JJ, Shearman JD, Robson KJ. Global prevalence of putative haemochromatosis mutations. J Med Genet 1997;34:275.

6. Andrews NC. Disorders of iron metabolism. N Engl J Med 1999;341:19861995.

7. Andrews NC. Forging a field: the golden age of iron biology. Blood 2008; 112:219-230

8. Olynyk JK, Trinder D, Ramm GA, Britton RS, Bacon BR. Hereditary hemochromatosis in the post-HFE era. Hepatology 2008;48:991-1001.

9. Gehrke SG, Kulaksiz H, Herrmann T, Riedel HD, Bents K, Veltkamp C, Stremmel W. Expression of hepcidin in hereditary hemochromatosis: evidence for a regulation in response to the serum transferrin saturation and to non-transferrin-bound iron. Blood 2003;102:371-376.

10. Olynyk JK, Cullen DJ, Aquilia S, Rossi E, Summerville L, Powell LW. A population-based study of the clinical expression of the hemochromatosis gene. N Engl J Med 1999;341:718-724.

11. Powell LW, Dixon JL, Ramm GA, Purdie DM, Lincoln DJ, Anderson GJ, Subramaniam VN, Hewett DG, Searle JW, Fletcher LM, Crawford DH, Rodgers H, Allen KJ, Cavanaugh JA, Bassett ML. Screening for hemochromatosis in asymptomatic subjects with or without a family history. Arch Intern Med 2006;166:294-301.

12. Allen KJ, Gurrin LC, Constantine CC, et al. Iron-overload-related disease in HFE hereditary hemochromatosis. N Engl J Med 2008;358:221-230.

13. Bacon BR, Britton RS. Clinical penetrance of hereditary hemochromatosis. N Engl J Med 2008;358:291-292.

14. Moirand R, Adams PC, Bicheler V, Brissot P, Deugnier Y. Clinical features of genetic hemochromatosis in women compared with men. Ann Intern Med 1997; 127:105-110

15. Wojcik JP, Speechley MR, Kertesz AE, Chakrabarti S, Adams PC. Natural history of $\mathrm{C} 282 \mathrm{Y}$ homozygotes for hemochromatosis. Can J Gastroenterol 2002;16:297-302.

16. Wood MJ, Powell LW, Ramm GA. Environmental and genetic modifiers of the progression to fibrosis and cirrhosis in hemochromatosis. Blood 2008; 111:4456-4462.
17. Tavill AS. American Association for the Study of Liver Diseases; American College of Gastroenterology; American Gastroenterological Association. Diagnosis and management of hemochromatosis. Hepatology 2001;33:1321-1328.

18. Kowdley KV, Tait JF, Bennett RL, Motulsky AG. GeneReviews. Available at: http://www.ncbi.nlm.nih.gov/bookshelf/br.fcgi?book=gene \& part $=$ hemochromatosis\#nemochromatosis.grID2784. Accessed November 10, 2008

19. McDermott JH, Walsh $\mathrm{CH}$. Hypogonadism in hereditary hemochromatosis. $J$ Clin Endocrinol Metab 2005;90:2451-2455.

20. Niederau C, Fischer R, Sonnenberg A, Stremmel W, Trampisch HJ, Strohmeyer G. Survival and causes of death in cirrhotic and in noncirrhotic patients with primary hemochromatosis. N Engl J Med 1985;313:1256-1262.

1. Bradbear RA, Bain C, Siskind V, et al. Cohort study of internal malignancy in genetic hemochromatosis and other chronic nonalcoholic liver diseases. J Natl Cancer Inst 1985;75:81-84.

22. Strohmeyer G, Niederau C, Stremmel W. Survival and causes of death in hemochromatosis. Observations in 163 patients. Ann N Y Acad Sci 1988;526: 245-257.

23. Hsing AW, McLaughlin JK, Olsen JH, Mellemkjar L, Wacholder S, Fraumeni JF Jr. Cancer risk following primary hemochromatosis: a population-based cohort study in Denmark. Int J Cancer 1995;60:160-162.

24. Elmberg M, Hultcrantz R, Ekbom A, et al. Cancer risk in patients with hereditary hemochromatosis and in their first-degree relatives. Gastroenterology 2003;125:1733-1741.

25. Kowdley KV. Iron, hemochromatosis, and hepatocellular carcinoma. Gastroenterology 2004;127(suppl 1):S79-S86.

26. Harrison SA, Bacon BR. Relation of hemochromatosis with hepatocellular carcinoma: epidemiology, natural history, pathophysiology, screening, treatment, and prevention. Med Clin North Am 2005;89:391-409.

27. Beutler E, Felitti VJ, Koziol JA, Ho NJ, Gelbart T. Penetrance of the $845 \mathrm{G}$ to A (C282Y) HFE hereditary haemochromatosis mutation in the USA. Lancet 2002;359:211-218.

28. Beutler E. The HFE Cys282Tyr mutation as a necessary but not sufficient cause of clinical hereditary hemochromatosis. Blood 2003;101:3347-3350.

29. Waalen J, Felitti V, Gelbart T, Ho NJ, Beutler E. Prevalence of hemochromatosis-related symptoms among individuals with mutations in the HFE gene. Mayo Clin Proc 2002;77:522-530.

30. McCune CA, Ravine D, Carter K, et al. Iron loading and morbidity among relatives of HFE C282Y homozygotes identified either by population genetic testing or presenting as patients. Gut 2006;55:554-562.

31. Gochee PA, Powell LW, Cullen DJ, Du Sart D, Rossi E, Olynyk JK. A population-based study of the biochemical and clinical expression of the H63D hemochromatosis mutation. Gastroenterology 2002;122:646-651.

32. Phatak PD, Bonkovsky HL, Kowdley KV. Hereditary hemochromatosis: time for targeted screening. Ann Intern Med 2008;149:270-272.

33. Mura C, Raguenes O, Férec C. HFE mutations analysis in 711 hemochromatosis probands: evidence for $\mathrm{S} 65 \mathrm{C}$ implication in mild form of hemochromatosis. Blood 1999;93:2502-2505.

34. Morrison ED, Kowdley KV. Genetic liver disease in adults. Early recognition of the three most common causes. Postgrad Med 2000;107:147$152,155,158-159$

35. Whittington CA, Kowdley KV. Review article: haemochromatosis. Aliment Pharmacol Ther 2002;16:1963-1975.

36. McLaren CE, McLachlan GJ, Halliday JW, et al. Distribution of transferrin saturation in an Australian population: relevance to the early diagnosis of hemochromatosis. Gastroenterology 1998;114:543-549.

37. Olynyk JK, Hagan SE, Cullen DJ, Beilby J, Whittall DE. Evolution of untreated hereditary hemochromatosis in the Busselton population: a 17-year study. Mayo Clin Proc 2004;79:309-313.

38. Powell LW, Halliday JW, Cowlishaw JL. Relationship between serum ferritin and total body iron stores in idiopathic haemochromatosis. Gut 1978; $19: 538-542$.

39. Beutler E, Felitti V, Ho NJ, Gelbart T. Relationship of body iron stores to levels of serum ferritin, serum iron, unsaturated iron binding capacity and transferrin saturation in patients with iron storage disease. Acta Haematol 2002;107:145-149.

40. Morrison ED, Brandhagen DJ, Phatak PD, et al. Serum ferritin level predicts advanced hepatic fibrosis among U.S. patients with phenotypic hemochromatosis. Ann Intern Med 2003;138:627-633.

41. Beaton M, Guyader D, Deugnier Y, Moirand R, Chakrabarti S, Adams P. Noninvasive prediction of cirrhosis in $\mathrm{C} 282 \mathrm{Y}$-linked hemochromatosis. Hepatology 2002;36:673-678

42. Guyader D, Jacquelinet C, Moirand R, et al. Noninvasive prediction of fibrosis in C282Y homozygous hemochromatosis. Gastroenterology 1998; 115:929-936.

43. Waalen J, Felitti VJ, Gelbart T, Beutler E. Screening for hemochromatosis by measuring ferritin levels: a more effective approach. Blood 2008;111: $3373-3376$

44. McGrath JS, Deugnier Y, Moirand R, Jouanolle AM, Chakrabarti S, Adams PC. A nomogram to predict $\mathrm{C} 282 \mathrm{Y}$ hemochromatosis. $J$ Lab Clin Med 2002;140:6-8. 
45. Mendler MH, Turlin B, Moirand R, et al. Insulin resistance-associated hepatic iron overload. Gastroenterology 1999;117:1155-1163.

46. Olynyk JK, Gan E, Tan T. Predicting iron overload in hyperferritinemia. Clin Gastroenterol Hepatol 2009;7:359-362.

47. Fuchs J, Podda M, Packer L, Kaufmann R. Morbidity risk in HFE associated hereditary hemochromatosis C282Y heterozygotes. Toxicology 2002;180: $169-181$.

48. Walsh A, Dixon JL, Ramm GA, et al. The clinical relevance of compound heterozygosity for the $\mathrm{C} 282 \mathrm{Y}$ and H63D substitutions in hemochromatosis. Clin Gastroenterol Hepatol 2006;4:1403-1410.

49. St Pierre TG, Clark PR, Chua-Anusorn W. Measurement and mapping of liver iron concentrations using magnetic resonance imaging. Ann N Y Acad Sci $2005 ; 1054: 379-385$.

50. Powell LW, George DK, McDonnell SM, Kowdley KV. Diagnosis of hemochromatosis. Ann Intern Med 1998;129:925-931.

51. Gagné G, Reinharz D, Laflamme N, Adams PC, Rousseau F. Hereditary hemochromatosis screening: effect of mutation penetrance and prevalence on cost-effectiveness of testing algorithms. Clin Genet 2007;71: 46-58.

52. Brissot P, Troadec MB, Bardou-Jacquet E, et al. Current approach to hemochromatosis. Blood Rev 2008;22:195-210.

53. Niederau C, Fischer R, Pürschel A, Stremmel W, Häussinger D, Strohmeyer
G. Long-term survival in patients with hereditary hemochromatosis. Gastroenterology 1996;110:1107-1119.

54. Andersen RV, Tybjaerg-Hansen A, Appleyard M, Birgens H, Nordestgaard BG. Hemochromatosis mutations in the general population: iron overload progression rate. Blood 2004;103:2914-2919.

55. Milman N, Pedersen P, á Steig T, Byg KE, Graudal N, Fenger K. Clinically overt hereditary hemochromatosis in Denmark 1948-1985: epidemiology, factors of significance for long-term survival, and causes of death in 179 patients. Ann Hematol 2001;80:737-744

56. Adams PC, Deugnier Y, Moirand R, Brissot P. The relationship between iron overload, clinical symptoms, and age in 410 patients with genetic hemochromatosis. Hepatology 1997;25:162-166.

57. Adams PC, Speechley M, Kertesz AE. Long-term survival analysis in hereditary hemochromatosis. Gastroenterology 1991;101:368-372.

58. von Delius S, Lersch C, Schulte-Frohlinde E, et al. Hepatocellular carcinoma associated with hereditary hemochromatosis occurring in non-cirrhotic liver. Z Gastroenterol 2006;44:39-42.

59. Geier D, Hebert B, Potti A. Risk of primary non-hepatocellular malignancies in hereditary hemochromatosis. Anticancer Res 2002;22:3797- 3799.

60. Yamashita C, Adams PC. Natural history of the C282Y homozygote for the hemochromatosis gene (HFE) with a normal serum ferritin level. Clin Gastroenterol Hepatol 2003;1:388-391. 